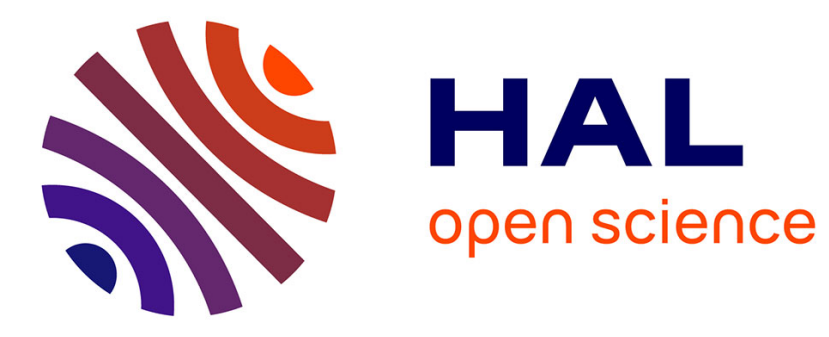

\title{
Les cultes isiaques et le pouvoir dans la Tétrapole syrienne \\ Julien Aliquot
}

\section{To cite this version:}

Julien Aliquot. Les cultes isiaques et le pouvoir dans la Tétrapole syrienne. Laurent Bricault; Miguel John Versluys. Power, politics, and the cults of Isis. Proceedings of the Vth International conference of Isis studies, Boulogne-sur-Mer, October 13-15, 2011, 180, E. J. Brill, pp.135-146, 2014, Religions in the Graeco-Roman World, 978-90-04-27827-1. 10.1163/9789004278271_007 . halshs-01709324

\section{HAL Id: halshs-01709324 \\ https://shs.hal.science/halshs-01709324}

Submitted on 1 Feb 2020

HAL is a multi-disciplinary open access archive for the deposit and dissemination of scientific research documents, whether they are published or not. The documents may come from teaching and research institutions in France or abroad, or from public or private research centers.
L'archive ouverte pluridisciplinaire HAL, est destinée au dépôt et à la diffusion de documents scientifiques de niveau recherche, publiés ou non, émanant des établissements d'enseignement et de recherche français ou étrangers, des laboratoires publics ou privés. 


\title{
Power, Politics and the Cults of Isis
}

Proceedings of the Vth International Conference of Isis

Studies, Boulogne-sur-Mer, October 13-15, 2011

(organised in cooperation with Jean-Louis Podvin)

\author{
Edited by
}

Laurent Bricault and Miguel John Versluys

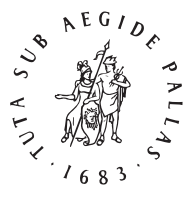

B R I L L

LEIDEN | BOSTON 


\section{Contents}

Preface vii

List of Figures viii

List of Participants xiii

Jean Leclant, in memoriam xiv

Laurent Bricault

\section{PART 1}

Isis and the longue durée

1 Isis and Empires 3

Laurent Bricault and Miguel John Versluys

2 Cuius regio, eius religio? Rulers and Religious Change in Greco-Roman Egypt $\quad 36$

Frederick Naerebout

3 Isis and the Evolution of Religions 62

Greg Woolf

PART 2

Isis and the Hellenistic World

4 Sarapis, Isis et le pouvoir lagide $\quad 95$

Bernard Legras

5 Les cultes isiaques dans l'espace séleucide $\quad{ }_{116}$

John Ma

6 Les cultes isiaques et le pouvoir dans la Tétrapole syrienne 135 Julien Aliquot

7 La reine Huldu et la coiffe isiaque. Isis et le pouvoir royal à Pétra ( I ${ }^{\text {er }}$ s. av. J.-C. -I ${ }^{\text {er }}$ s. apr. J.-C.) 147

Christian-Georges Schwentzel 
8 « Isis » et « Athènes » : épigraphie, espace et pouvoir à la basse époque hellénistique $\quad 163$

Paraskevi Martzavou

\section{PART 3}

Isis and the Roman World

9 Le basileion, les reines et Actium 195

Richard Veymiers

10 The Flavians: Pharaonic Kingship between Egypt and Rome

Giuseppina Capriotti Vittozzi

11 Les cultes isiaques et les pouvoirs locaux en Italie $\quad 260$ Valentino Gasparini

12 Le tropisme isiaque des Sévères : une acmé reconsidérée?

300 Jean-Louis Podvin

13 Gens isiaca et identité polythéiste à Rome à la fin du IV

s. apr. J.-C. $\quad 326$

Laurent Bricault

Index Nominum $\quad 361$ 


\title{
Les cultes isiaques et le pouvoir dans la Tétrapole syrienne
}

\author{
Julien Aliquot
}

À en croire les historiens d'Alexandre le Grand, les premiers contacts entre les Séleucides et les dieux de la vallée du Nil remonteraient au temps de la conquête de l'Orient par les Macédoniens. Arrien affirme tenir cette information des éphémérides royales dans son Anabase (VII, 26, 2). Plutarque évoque le même épisode dans sa Vie d'Alexandre (76). Tous deux rapportent qu'à Babylone, au mois de juin 323 av. J.-C., Séleucos, alors qu'il n'était encore que le compagnon du conquérant, aurait veillé dans l'attente d'une réponse de Sarapis, pour décider s'il était opportun ou non de transporter le roi à l'article de la mort jusqu'au temple du dieu. Qu'un oracle de Sarapis ou d'Osiris-Apis ait été consulté alors n'a rien d'impossible, car des prêtres égyptiens ont suivi l'armée macédonienne jusqu'en Babylonie et auraient encore été sollicités pour embaumer le corps d'Alexandre'. De même, que Séleucos ait rendu hommage aux dieux de l'Égypte à un moment ou à un autre de sa longue carrière est envisageable. Qu'il ait manipulé des objets de culte égyptiens est également possible. Une inscription de Didymes rappelle que le fondateur de la dynastie séleucide, devenu roi, avait fait don au sanctuaire d'Apollon d'une phiale peutêtre consacrée à Osiris, parmi les nombreux objets de luxe pris à Démétrios Poliorcète, en $288 / 7$ av. J.-C. ${ }^{2}$. Mais, même si la lecture du nom d'Osiris dans ce document était assurée, rien ne prouverait en définitive que Séleucos I $^{\mathrm{er}}$ ait eu

1 Quinte-Curce IV, 10, 4-7; X, 10, 9-13. Voir P. Goukowsky, Essai sur les origines du mythe d'Alexandre I (Nancy 1978) 199-200.

2 A. Rehm, Didyma II. Die Inschriften (Berlin 1958) nº 424. À la l. 33, la lecture $\alpha \lambda \lambda \eta \eta(s c i l . ~ \varphi ı ́ \alpha \lambda \eta)$

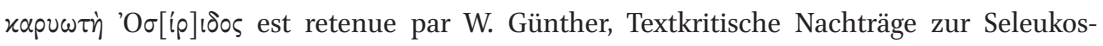
Stiftung in Didyma, Istanbuler Mitteilungen 27-28 (1977-1978) 263 (J. et L. Robert, Bull. ép. [1980] no 458), puis par G. Petzl, Epigraphische Nachlese, EA 13 (1989) 131-133, et en dernier lieu dans le RICIS $n^{\circ}$ 304/1101. Cependant, la découverte de nouvelles copies a amené G. Petzl, Zum Brief Seleukos' I. C.B. Welles, Royal Correspondence Nr. 5 (Griechische Inschriften aus dem Nachlass G. Cupers [III]), dans : H. Malay (éd.), Erol Atalay Memorial (Izmir 1991) 145-152

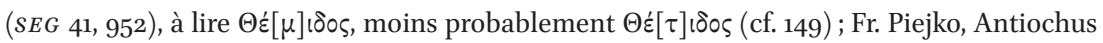
III and Ilium, APF 37 (1991) 38 n. 9o, propose de façon peu convaincante Oự[ $\pi]$ b étant une hypostase d'Artémis. Voir aussi la contribution de J. Ma, dans ce volume, p. 117-122. 
un quelconque intérêt pour les divinités égyptiennes. Comme on le verra, la même conclusion semble s'imposer à propos de ses successeurs.

Si l'on prend en considération les régions du Proche-Orient que les Séleucides ont dominées entre la fin du IV ${ }^{\mathrm{e}}$ siècle et le début du Ier siècle avant le début de l'ère chrétienne, force est de constater à nouveau que, dans l'état actuel de nos connaissances, les cultes isiaques n'ont rencontré ici qu'un succès très modeste. Deux cas font figure d'exception. Celui de la partie de la Syrie qui s'étend au sud de l'Éleuthéros ne nous occupera pas ici : il concerne une région que les Ptolémées ont occupée durant tout le III ${ }^{\mathrm{e}}$ siècle av. J.-C., dont les relations avec l'Égypte sont anciennes et traditionnelles et où la présence de sanctuaires isiaques, à Byblos et à Tyr, correspond à un héritage antérieur aux Séleucides ${ }^{3}$. Le cas de la Tétrapole syrienne retiendra davantage notre attention, à travers la documentation relative à Antioche sur l'Oronte, Séleucie de Piérie et Laodicée-sur-mer (Apamée de Syrie n'ayant rien livré sur le sujet qui nous intéresse). C'est là, au cœur du domaine séleucide, que l'on peut le mieux appréhender la question des rapports entre les cultes isiaques et les pouvoirs royaux et civiques. Les témoignages utiles à la discussion remontent pour la plupart à l'époque romaine, période au cours de laquelle les cultes isiaques ont connu une diffusion sans précédent dans le bassin méditerranéen, période pour laquelle la question de leur diffusion en Syrie ne se pose plus dans les mêmes termes qu'au temps de la rivalité entre Séleucides et Lagides. De manière remarquable et inattendue, ces témoignages nous ramèneront cependant à la guerre laodicéenne et à la troisième guerre de Syrie, qui opposa Séleucos II Callinicos (246-226/5) à Ptolémée III Évergète (246-222) de 246 à 241 av. J.-C.

\section{Antioche sur l'Oronte}

Dans l'Antiochikos, discours prononcé en 356 apr. J.-C. à l'occasion de la célébration des jeux olympiques locaux, Libanios cherche à mettre en valeur l'idée que les dieux ont de tout temps été irrésistiblement attirés vers Antioche, de sorte que la cité, qui était aussi la patrie du rhéteur, pouvait rivaliser avec l'Olympe. C'est ainsi qu'Isis aurait voyagé de Memphis vers la grande métropole syrienne sous le règne de Séleucos II, le quatrième souverain de la dynastie

3 L. Bricault, Atlas de la diffusion des cultes isiaques (Paris 2001) 70-77; J. Aliquot, Aegyptiaca et isiaca de la Phénicie et du Liban aux époques hellénistique et romaine, Syria 81 (2004) 201228 ; id., La vie religieuse au Liban sous l'Empire romain (Beyrouth 2009) 171-183. Sur Byblos, voir aussi L. Bricault, Poids de Byblos inscrits au basileion, dans : L. Bricault, R. Veymiers (éd.), Bibliotheca Isiaca II (Bordeaux 2011) 137-143. 
séleucide. Voici le passage de l'Antiochikos (114) en question : «En effet, Isis, la statue cornue d'Égypte, quitta Memphis et émigra ici, après avoir incité en rêve Séleucos (le quatrième à partir de Séleucos) à faire appel à elle, ayant incité de même Ptolémée à faire promptement don de la déesse ; les navires parés,

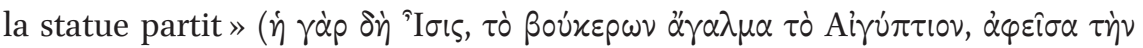

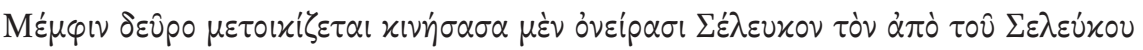

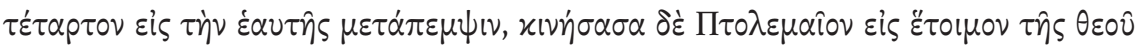

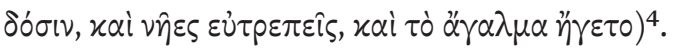

On a déduit du témoignage de Libanios que Séleucos II aurait institué à Antioche le culte de la déesse égyptienne, en général pour en douter aussitôt ${ }^{5}$. Il est vrai qu'aucun document ne confirme une telle innovation, ni de la part de ce roi, ni de la part de ses prédécesseurs et de ses successeurs. Le monnayage royal des Séleucides atteste bien l'usage de l'imagerie isiaque, mais essentiellement à des fins commémoratives et seulement à partir d'Antiochos IV Épiphane, d'abord pour célébrer la réussite de ce dernier dans l'invasion de l'Égypte, en 169 et en 168 av. J.-C. ${ }^{6}$, puis à nouveau sous Alexandre I ${ }^{\text {er }}$ Balas,

4 Libanios, Antiochikos (Oratio XI), éd. R. Foerster, Libanii opera I, 2. Orationes VI-XI (Leipzig 1903) 473 (ma traduction). G. Downey, Libanius' Oration in Praise of Antioch (Oration XI), PAPhS 103 (1959) 652-686, ne commente pas ce passage, si ce n'est pour identifier le souverain séleucide à Séleucos II (682); id., A History of Antioch in Syria (Princeton 1961) 91-92, est allusivement sceptique à l'égard du témoignage de Libanios. A.F. Norman, Antioch as a Centre of Hellenic Culture as Observed by Libanius (Liverpool 2000) 28 n. 56, partageant la même défiance, en a pressenti l'intérêt : « Seleucus II Callinicus (246-226 вС) lost the Seleucid lands in Asia Minor and in the East. The divine support for Antioch is used to veil such secular reverses, even if the story is not an invention of Libanius himself to account for the growing popularity of the cult of Isis. »

5 Voir en dernier lieu SNRIS 155-156 et n. 403. Fr. Dunand, Le culte d'Isis dans le bassin oriental de la Méditerranée III (Leyde 1973) 123, exprimait les mêmes doutes, tout en identifiant à tort le souverain séleucide mentionné par Libanios à Séleucos IV. À propos d'Antioche, rappelons pour mémoire l'article de F.W. Norris, Isis, Sarapis and Demeter in Antioch of Syria, HThR 75 (1982) 189-207, qui englobe abusivement Antioche et Séleucie de Piérie dans le même ensemble et qui fourmille d'erreurs : notamment, l'auteur (190) n'a pas pris garde au fait que les dates indiquées dans le catalogue numismatique du British Museum pouvaient être données selon le comput séleucide et non dans le calendrier julien, ce qui le conduit à dater la première monnaie d'Antioche portant l'image du basileion en 227 av. J.-C. ; il s'agit en réalité d'une monnaie de l'an 86/5 av. J.-C. Voir, dans ce volume, la contribution de R. Veymiers, p. 210-212.

6 Sur les bronzes égyptisants d'Antiochos IV frappés à Antioche, voir G. Le Rider, Antiochos IV (175-164) et le monnayage de bronze séleucide, $B C H 118$ (1994) 17-20 ; id., Antioche de Syrie sous les Séleucides (Paris 1999) 223-224 et 231-232 ; SNRIS 155; Seleucid Coins II, 44-45, 49 et 68-69 $\mathrm{n}^{\text {os }}$ 1412-1415. L'hypothèse de l'émission commémorative remonte à E.T. Newell, The Seleucid mint of Antioch (Chicago 1978) 24-27. 
Antiochos VI, Antiochos VII et Antiochos VIII, pour fêter leurs unions ou rappeler leur filiation avec des princesses lagides, en premier lieu avec Cléopatra Théa ${ }^{7}$. À Antioche, il faut attendre l'année 92/1 av. J.-C. pour voir apparaître des monnaies civiques portant le basileion; encore ne s'agit-il que d'une marque monétaire située dans le champ du revers, en marge des divinités honorées (Zeus, Tyché), et substituable à des monogrammes et à d'autres symboles (palme, caducée, corne d'abondance, étoile, croissant) dont la signification religieuse est loin d'être assurée ${ }^{8}$. Rien ne porte donc à croire que les cultes isiaques aient eu une grande importance, ni chez les Séleucides, ni à Antioche à l'époque hellénistique ${ }^{9}$.

Si l'on suit Libanios à la lettre, il semble du reste improbable que Séleucos II ait institué le culte d'Isis à Antioche. Il convient de prendre en considération tout le passage de l'Antiochikos qui se rapporte à l'arrivée de la déesse en Syrie et non seulement l'extrait concernant le souverain séleucide. Dès lors, le rôle de Ptolémée III apparaît au moins aussi important que celui de Séleucos II. On peut se demander si l'institution du culte d'Isis à Antioche n'est pas survenue à l'occasion d'une entente entre les deux souverains, par exemple lors de la paix de 241 av. J.-C., qui marque la fin de la troisième guerre de Syrie. Le règlement d'un conflit entre deux adversaires politiques à la suite de l'intervention en songe d'un dieu égyptien est bien illustré par une inscription de Thessalonique ${ }^{10}$. Le texte, gravé au début de l'époque impériale, pourrait reproduire une inscription plus ancienne. Il évoque l'introduction du culte de Sarapis à Oponte, en Locride orientale, par un certain Xénainétos, à qui le dieu avait ordonné de demander à Eurynomos, un concitoyen avec qui il

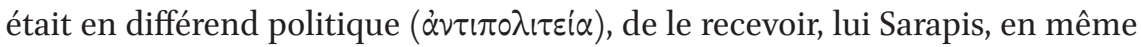
temps que sa sœur Isis. Xénainétos devait transmettre à Eurynomos une lettre

SNRIS 156 ; Seleucid Coins II, 211-212, $245 \mathrm{n}^{\circ} 1846$ (Alexandre I ${ }^{\text {er }}$ Balas), $330 \mathrm{n}^{\circ} 2021$ (Antiochos VI ou VIII), 353, 363, 366-368 $\mathrm{n}^{\text {os }}$ 2066-2067, 369-370 $\mathrm{n}^{\text {os }} 2071-2072,393 \mathrm{n}^{\text {os }} 2125 \mathrm{~A}$ 2126 (Antiochos VII), 470, 479-480 nos 2273-2274 (Cléopatre Théa et Antiochos VIII). Les exemplaires connus forment des séries datées de $92 / 1$ à 86/5, puis de $47 / 6$ à $42 / 1$ et enfin de 30/29 à 25/4 av. J.-C. Voir K. Butcher, Coinage in Roman Syria (Londres 2004) 307-312, 315-316 et 318-319; R. McAlee, The Coins of Roman Antioch (Lancaster-Londres 2007) $74 \mathrm{n}^{\text {os }} 47$ et $49,78 \mathrm{n}^{\text {os }} 68 \mathrm{~b}$, $70 \mathrm{cot}$ et $7 \mathrm{oB}$.

$9 \quad$ Notons au passage que la même conclusion paraît valable sous l'Empire romain dans le cas d'Antioche, les éléments réunis par F.W. Norris (cité supra n. 5) ne témoignant au mieux que de dévotions privées (fragment de statuaire, lampes, anthroponyme isiaque isolé, mosaïques d'interprétation controversée) et de la piété des empereurs (sacrifices de Julien, monnaies impériales frappées par l'atelier d'Antioche aux III et $\mathrm{IV}^{\mathrm{e}}$ siècles apr. J.-C.).

RICIS $\mathrm{n}^{\circ}$ 113/0536. Je remercie P. Martzavou d'avoir attiré mon attention sur ce texte. 
trouvée sous son oreiller. Il la trouva effectivement après un second songe et la transmit à son ancien ennemi, qui fut convaincu de recevoir les dieux égyptiens et de leur offrir des sacrifices.

Le parallèle entre le récit de l'introduction du culte de Sarapis à Oponte et celui de l'arrivée d'Isis à Antioche est si frappant que l'on peut supposer que Libanios se réfère à un modèle tiré de l'immense tradition de l'onirisme grec et égyptien. Pour que le parallèle soit exact, il faudrait toutefois que ce fût Ptolémée III et non Séleucos II qui eût accepté de recevoir la statue d'Isis, ce qui serait absurde. Quand bien même on admettrait cette hypothèse, l'intervention de Séleucos II n'en serait pas moins douteuse et artificielle. Le parti pris pro-séleucide relève en effet du système dans l'Antiochikos. Il conduit Libanios à refuser aux Lagides toute initiative heureuse dans leurs entreprises politiques et religieuses: Ptolémée I ${ }^{\mathrm{er}}$ ne doit de régner qu’à la décision de Séleucos ${ }^{\mathrm{er}}$ (82); plus tard, sous Antiochos II, la statue d'Artémis, que Ptolémée II avait souhaité emporter en Égypte, choisit finalement de rentrer chez elle (109). L'apparition providentielle d'Isis en songe et l'appel de Séleucos II apparaissent également comme des motifs conventionnels et surtout utiles à l'argumentation de Libanios. En revanche, il est clair, d'une part, qu'il est bien question d'une statue de culte représentant la déesse ( $\left.\alpha \gamma^{\prime} \alpha \lambda \mu \alpha\right)$ et, d'autre part, que l'arri-

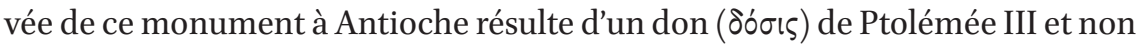
de Séleucos II, son rival dans la troisième guerre de Syrie.

La seule période propice à l'envoi de la statue d'Isis me parait donc être celle de l'occupation lagide d'Antioche, entre 246 et 244 av. J.-C. environ. Le papyrus de Gourob, qui reproduit la relation de l'expédition par Ptolémée III, retrace le début des événements ${ }^{11}$. En dépit de son caractère fragmentaire, ce document indique que le souverain lagide, accueilli avec ferveur à Antioche, avait eu le temps de participer à des sacrifices et de tenir conseil auprès des autorités de la cité, avant que des troubles ne le rappellent en Égypte. L'institution du culte d'Isis à Antioche a pu survenir dans ce laps de temps. Elle correspondrait à ce que l'on sait de l'attitude religieuse de Ptolémée III.

Les découvertes du Sérapéum d'Alexandrie, sur la colline de Rhacôtis, identifient Ptolémée III au (ou plutôt à l'un des principaux) fondateur(s) de ce sanctuaire ${ }^{12}$. Le roi a également patronné la construction de nombreux

11 J.P. Mahaffy, J.G. Smyly, The Flinders Petrie Papyri (Dublin 1891-1905) II no 45 et III no 144, texte révisé et commenté par M. Holleaux, Études d'épigraphie et d'histoire grecques III (Paris 1942) 281-310. Cf. Fr. Piejko, Episodes from the Third Syrian War in a Gurob Papyrus, 246 BC, AFP 36 (1990) 13-27 ; M. Sartre, D’Alexandre à Zénobie (Paris 2001) 193-194.

12 Plaquettes de fondation: A. Rowe, Discovery of the famous temple and enclosure of Serapis at Alexandria (Annales du Service des Antiquités de l'Égypte, Suppl. 2) (Le Caire 1946); 
édifices religieux en Égypte, notamment à Canope et à Edfou ${ }^{13}$. Il n'en est pas resté là. D'après le Commentaire sur Daniel (XI, 7-9) de saint Jérôme, il avait constitué un important butin de guerre lors de l'expédition qu'il avait conduite en Orient après la prise d'Antioche. Poussant jusqu'à Babylone, il en avait aussi profité pour récupérer les statues des dieux égyptiens emportées par les Perses sous le règne de Cambyse, en 525-522 av. J.-C. ${ }^{14}$. Le nombre de deux mille cinq cents, donné par Jérôme, est peut-être exagéré. L'inscription d'Adoulis (245-243 av. J.-C.), le décret de Canope (238 av. J.-C.) et celui de Raphia (217 av. J.-C.) n'en célèbrent pas moins le rôle de Ptolémée III dans le rapatriement des statues vers leurs sanctuaires ${ }^{15}$. Ces témoignages avaient bien sûr pour objectif premier de renouveler solennellement la confiance du clergé traditionnel envers le souverain. Mais la publicité donnée aux prises de guerre faites sur les terres de Séleucos II et au retour des dieux égyptiens dans leur pays d'origine plaçait aussi le roi lagide dans les pas d'Alexandre le Grand tout en discréditant les Séleucides assimilés aux Perses. La fondation (vraisemblablement sans lendemain) d'un sanctuaire isiaque à Antioche, dans la ville qui avait constitué jusqu'alors le centre du pouvoir séleucide et qui se trouvait occupée en 246

P. Jouguet, Les dépôts de fondation du temple de Sarapis à Alexandrie, CRAI (1946) 680687 ; É. Bernand, Inscriptions grecques d'Alexandrie ptolémaïque (Le Caire 2001) $42-43 \mathrm{n}^{\circ} 13$. Architecture et archéologie : J.S. McKenzie, Sh. Gibson, A.T. Reyes, Reconstructing the Serapeum in Alexandria from the archaeological evidence, JRS 94 (2004) 73-121, en particulier 81-84; M. Sabottka, Das Serapeum in Alexandria (Le Caire 2008) 67-245.

13 A. Bernand, Le Delta égyptien d'après les textes grecs I (Le Caire 1970) $236-237 \mathrm{n}^{\circ} 7$ (sanctuaire d'Osiris à Canope); S. Cauville, D. Devauchelle, Le temple d'Edfou : étapes de la construction, nouvelles données historiques, Revue d'égyptologie 35 (1984) 31-32 (naos d'Horus à Edfou). Voir aussi le texte de fondation par Ptolémée III d'un Sérapéum, que Ph. Borgeaud, Y. Volokhine, Plaquette d'argent épigraphe provenant du dépôt de fondation d'un Sérapeum, dans : J.-L. Chappaz, S. Vuillemier (éd.), « Sortir au jour ». Art égyptien de la Fondation Martin Bodmer (Genève 2001) 151-156, proposent de localiser à Canope. L. Bricault, Isis, Dame des flots (Liège 2006) 31-33, reprend cette hypothèse. Pour sa part, J. Bingen, Bull. ép. (2002) no 505, exprime des doutes sur l'authenticité du monument inscrit.

14 P. Briant, Histoire de l'Empire perse (Paris 1996) 66-72 et 915-916, sur la folie et l'impiété de Cambyse.

15 D. Devauchelle, Le sentiment anti-perse chez les anciens Égyptiens, Transeuphratène 9 (1995) 71-72 ; K. Winnicki, Carrying off and bringing home the statues of the gods. On an aspect of the religious policy of the Ptolemies towards the Egyptians, JJP 24 (1996) 149-190 ; P. Briant, Quand les rois écrivent l'histoire : la domination achéménide vue à travers les inscriptions officielles lagides, dans : N. Grimal, M. Baud (éd.), Événement, récit, histoire officielle. Écriture de l'histoire dans les monarchies antiques (Paris 2003) 173-186; S. Pfeiffer, Das Dekret von Kanopos (Leipzig 2004) 20, 84-88. 
av. J.-C., ressortirait donc à la politique édilitaire et religieuse de Ptolémée III. C'est ce que Libanios ou sa source aurait contribué à dissimuler en peuplant les rêves de Séleucos II de la figure d'une Isis cornue.

\section{Séleucie de Piérie}

Le célèbre excursus que Tacite consacre à Sarapis dans ses Histoires rappelle, sur un ton dubitatif, une historiette intrigante en marge de la tradition relative aux origines du type iconographique du dieu $(\mathrm{IV}, 84,4)$ : «je n'ignore pas non plus que certains le font venir de Séleucie, ville de Syrie, sous le règne du Ptolémée qui appartenait à la troisième génération », c'est-à-dire sous Ptolémée III Évergète (nec sum ignarus esse quosdam qui Seleucia urbe Syriae accitum regnante Ptolemaeo, quem tertia aetas tulit ${ }^{16}$. Clément d'Alexandrie revient sur le même épisode dans le Protreptique (IV, 48, 3), véritable « exhortation » visant à démontrer la supériorité du christianisme sur les cultes païens. Le Père de l'Église précise dans quelles circonstances la statue cultuelle de Sarapis a voyagé : «Isidôros est seul à dire que la statue fut amenée de Séleucie près d'Antioche, parce que les habitants de cette ville, s'étant eux aussi trouvés dans

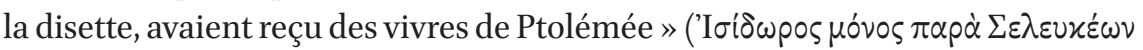

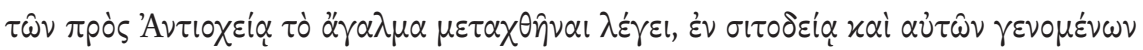

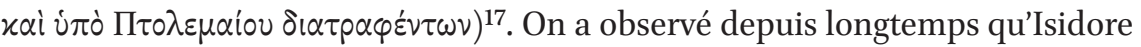
de Charax, historien et géographe contemporain de Strabon, était la source probable de cette tradition ${ }^{18}$. En revanche, personne à ma connaissance n'a proposé d'interprétation satisfaisante aux témoignages croisés de Tacite et de Clément d'Alexandrie à propos de Séleucie. Lorsqu'ils ne se sont pas mépris sur l'identification de la ville à laquelle ces auteurs font allusion ${ }^{19}$, les commentateurs se sont généralement concentrés sur la question de l'image prototypique

\footnotetext{
16 Tacite, Histoires III. Livres IV et V, éd. et trad. H. Le Bonniec (Paris 2003) 73.

17 Clément d'Alexandrie, Le Protreptique, éd. et trad. Cl. Mondésert et A. Plassart (Paris 2004) 109 .

18 Ainsi déjà A. Bouché-Leclercq, La politique religieuse de Ptolémée Soter et le culte de Sérapis, RHR 46 (1902) 14 n. 1.

19 L. Robert, Hellenica XI-XII (Paris 1960) 87 n. 1, identifie à tort la Séleucie de Tacite à Séleucie du Tigre, ville de Mésopotamie et non de Syrie, alors qu'E. Bikerman, Institutions des Séleucides (Paris 1938) 251, auquel il renvoie, ne précise pas de quelle cité il s'agit. L'erreur s'est perpétuée dans l'IBIS $\mathrm{n}^{\mathrm{o}} 1235$ et encore chez. Ph. Borgeaud et Y. Volokhine, La formation de la légende de Sarapis : une approche transculturelle, $A R G 2$ (2000) 59 n. 94.
} 
de Sarapis et sur le rôle des souverains lagides dans ce domaine ${ }^{20}$. L'intérêt des deux passages cités me semble être ailleurs.

Il faut à nouveau se remémorer les événements de la troisième guerre de Syrie. Au Proche-Orient, la conséquence la plus remarquable du conflit consiste en l'occupation de Séleucie de Piérie par les Lagides de 246 à 219 av. J.-C. ${ }^{21}$. Les Ptolémées tiennent ainsi la cité qui servait de débouché maritime à Antioche, menaçant le cœur du royaume séleucide pendant presque trente ans : «sans parler du déshonneur que causait présentement à la royauté l'occupation de la place par les rois d'Égypte », comme l'écrit Polybe $(\mathrm{V}, 58,5-7)$ à propos du projet de reconquête entrepris sous Antiochos III, «elle offrait sous le rapport des opérations les plus grands et les plus précieux avantages; tant qu'elle était au pouvoir de l'ennemi, elle opposait le plus grand obstacle à toutes leurs entreprises (celles des Séleucides); quel que fût leur plan d'invasion, leur propre pays n'exigerait pas moins de mesure de prévoyance et de défense, à cause du danger qui venait de ce côté ». Dans ces conditions, il paraît probable que des Grecs d'Égypte et des Égyptiens hellénisés se soient établis et qu'ils aient fondé à Séleucie un sanctuaire de Sarapis, avec ou sans le concours des citoyens de souche qui n'avaient pas fui à Antioche et de mercenaires étrangers.

Il n'est pas certain que ce sanctuaire soit celui qui a été fouillé sur le site de Séleucie dans les années 1930 par l'université de Princeton et dont l'attribution ne repose que sur la découverte d'une statuette d'Isis dans les ruines

20 Selon Bouché-Leclercq, Politique religieuse de Ptolémée Soter 20 et 25, la statue de Séleucie aurait fait partie du butin de guerre de Ptolémée III et l'histoire de la famine de la ville serait une invention. Fr. Dunand, Le culte d'Isis dans le bassin oriental de la Méditerranée I (Leyde 1973) 58, reprenant l'idée de S. Reinach, Le moulage des statues et le Sérapis de Bryaxis, $R A$ (1902) II, 20-21, et d'I. Lévy, Sarapis, $R H R 61$ (1910) 145 et 176, rappelle que Bryaxis, le sculpteur du IV ${ }^{\mathrm{e}}$ siècle av. J.-C. parfois crédité de l'invention de l'idole de Sarapis, a été actif dans la région d'Antioche à la fin de sa vie, et suppose que « Ptolémée III, ayant occupé Séleucie, a pu y trouver une statue dans le style de Bryaxis qu'il aurait ramenée en Égypte ». W. Hornbostel, Sarapis (Leyde 1973) 43 n. 4, juge cette théorie étrange, mais ne cherche pas à expliquer pourquoi Séleucie est citée. Borgeaud, Volokhine, La légende de Sarapis 46 n. 36 , se contentent eux aussi de relever la mention de la ville chez Tacite et Clément d'Alexandrie. N. Belayche, Le possible « corps » des dieux : retour sur Sarapis, dans: Fr. Prescendi, Y. Volokhine (éd.), Dans le laboratoire de l'historien des religions. Mélanges offerts à Philippe Borgeaud (Genève 2011) 227-250, étudie la fabrication de l'idole de Sarapis d'après Clément d'Alexandrie et Origène, sans s'attarder non plus sur la tradition qui fait venir la statue de Syrie.

21 É. Will, Histoire politique du monde hellénistique (Nancy 1979-1982) I, 259, et II, 30 ; Sartre, D’Alexandre à Zénobie 195. 
du temple ${ }^{22}$. Rappelons aussi qu'une statue de marbre en ronde bosse représentant Sarapis trônant avec Cerbère à ses pieds a été retrouvée aux environs de la ville en $1954^{23}$. Le monument est conservé au musée d'Antakya. Sa provenance exacte est indéterminée et il n'est pas sûr qu'il s'agisse d'une statue de culte. Sa datation de l'époque romaine laisse au moins supposer que le dieu a continué d'être honoré à Séleucie après le départ des Lagides, au moins sous une forme privée. Plus sûrement, on remarquera que la cité, à l'époque où elle était défendue par les Ptolémées, était susceptible de pâtir de sa situation assez enclavée, dès lors qu'elle se trouvait détachée de sa voisine et des ressources de la plaine de l'Amykè (Amuq). En somme, l'anecdote que rapportent Tacite et Clément d'Alexandrie serait née d'un fait bien réel et certainement indépendant de la création du type iconographique de Sarapis: le présent d'une statue de culte $(\not \alpha \gamma \alpha \lambda \mu \alpha)$, fait par les habitants de Séleucie de Piérie à Ptolémée III pour remercier leur royal bienfaiteur de les avoir sauvés de la famine.

\section{Laodicée-sur-mer}

Le cas de Laodicée-sur-mer illustre les rapports entre les propriétaires d'un sanctuaire privé de Sarapis et d'Isis, le pouvoir séleucide, représenté par l'épistate du roi, et les autorités d'une cité grecque de la côte syrienne. Il est documenté par la copie romaine d'un décret hellénistique daté en janvier 174 av. J.-C., sous le règne d'Antiochos $\mathrm{IV}^{24}$. L'inscription révèle que les péliganes, c'est-à-dire les membres du conseil civique de Laodicée-sur-mer, ont été appelés à se prononcer sur la question des droits dont les fidèles devaient

22 R. Stillwell (éd.), Antioch on-the-Orontes III. The Excavations 1937-1939 (Princeton 1941) 32-34, fig. 39-42 (temple), et 124, pl. 16 no 365 (statuette).

23 S. Keskil, Hatay Hadesi, Türk Arkeoloji Dergisi 12/2 (1963) 88-9o. Parallèles listés dans V. Tran tam Tinh, Le culte des divinités orientales en Campanie (Leyde 1972) 50-51 n. 1.

24 D’abord publié par P. Roussel, Décret des péliganes de Laodicée-sur-mer, Syria 23 (19421943) 21-32, d'après le relevé et l'estampage du Père R. Mouterde, le texte a été repris par ce dernier dans les Inscriptions grecques et latines de la Syrie IV. Laodicée, Apamène, $n^{\text {os }}$ 1243-1997 (Paris 1953) no 1261, puis par G. Klaffenbach, Epigraphische Studien, Philologus 97 (1948) 376-379 (J. et L. Robert, Bull. ép. [1950] no 208), dans le RICIS no 402/0301, et enfin par J.D. Sosin, Unwelcome dedications : public law and private religion in Hellenistic Laodicea by the sea, $C Q 55$ (2005) 130-139. Voir aussi, sur sa regravure, H. Seyrig, Poids antiques de la Syrie et de la Phénicie sous la domination grecque et romaine, Bulletin du Musée de Beyrouth 8 (1946-1948) 67. La stèle sur laquelle il est inscrit est conservée au Musée national de Beyrouth. J'en donnerai une nouvelle édition dans le catalogue épigraphique de ce musée (en préparation avec J.-B. Yon). 


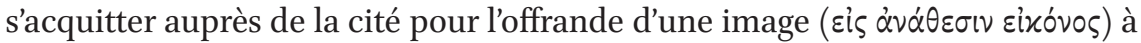
l'intérieur de l'espace consacré aux dieux égyptiens. Les commentaires étendus de P. Roussel, G. Klaffenbach et L. Robert ont été récemment complétés avec profit par J.D. Sosin. Ils me dispensent provisoirement de reprendre tout le dossier. Retenons que le décret des péliganes, en dépit de son caractère exceptionnel, n'indique aucunement que Sarapis et Isis avaient une foule de fidèles à Laodicée-sur-mer sous Antiochos IV. Ce document concerne plutôt un petit sanctuaire privé dont la situation en vue aurait incité les habitants de la ville à y ériger tant de monuments (statues honorifiques, images diverses) que la sécurité des lieux s'en serait trouvée menacée (le terme utilisé, gixwóv, doit être distingué de celui qui désigne le monument sculpté qui fait l'objet d'un culte, $\alpha$ $\gamma \alpha \lambda \mu \alpha$, à Antioche et à Séleucie, par exemple). En conséquence, les autorités de la cité, suivant la proposition de l'épistate Asclépiadès, ont pris des mesures afin d'empêcher la destruction prévisible des bâtiments cultuels, tout en récupérant les revenus de la taxe sur l'érection des statues, qui échappaient jusqu'alors à la cité en raison du caractère privé du sanctuaire.

Une question reste posée, celle des circonstances dans lesquelles le sanctuaire isiaque de Laodicée-sur-mer a été fondé. Hôros, Apollodôros et Antiochos, les propriétaires du lieu, prêtres de Sarapis et d'Isis et probablement frères, appartiennent manifestement à une famille d'Égyptiens hellénisés ou de Grecs d'Égypte établis en Syrie depuis longtemps. `̊pos, le nom théophore du premier d'entre eux, est révélateur de leur origine. Les trois prêtres précisent par ailleurs qu'ils possèdent le sanctuaire et l'îlot sur lequel le sanctuaire est bâti avec les fils d'Apollodôros, leurs cousins descendant du même aïeul. En d'autres termes, tous sont parents par un ou par plusieurs ancêtres communs depuis deux générations (de même qu'à Délos, un certain Apollonios, prêtre de Sarapis, affirme que les biens consacrés au dieu appartiennent à sa famille depuis deux générations ${ }^{25}$ ). Il semble ainsi probable que l'institution du culte de Sarapis et d'Isis remonte au dernier tiers du IIIe siècle av. J.-C. Or, à cette époque, la cité était vraisemblablement sous domination lagide.

Après avoir été le principal atelier de production des alexandres séleucides dans la première moitié du III siècle av.J.-C., Laodicée-sur-mer a cessé de battre monnaie pour le compte des Séleucides vers le milieu des années 240 av. J.-C. De façon convaincante, A. Houghton a lié l'interruption de l'activité monétaire de la ville aux succès militaires de Ptolémée III au début de la troisième guerre de Syrie. Il en a tiré la conclusion que les Lagides étaient parvenus à s'emparer 
de Laodicée-sur-mer ${ }^{26}$. Il est impossible de préciser combien de temps cette occupation a duré dans l'état actuel de nos connaissances. L'exemple des villes voisines de Séleucie de Piérie ${ }^{27}$, de Posideion ${ }^{28}$ et d'Héraclée-sur-mer ${ }^{29}$ laisse cependant supposer que la cité est restée de manière durable aux mains des Ptolémées, peut-être jusqu'au règne d'Antiochos III $(223-187)^{30}$, le souverain qui parvint à reconquérir définitivement les territoires des Lagides en Syrie, en tout cas assez longtemps pour justifier l'immigration des fondateurs du sanctuaire isiaque local.

\section{Conclusion}

Rien ne porte à croire que les Ptolémées auraient fait délibérément œuvre de propagande en faveur de Sarapis et d'Isis, les patrons de leur dynastie et les divinités tutélaires d'Alexandrie, leur capitale ${ }^{31}$. Néanmoins, l'établissement de la domination lagide sur certaines régions du Proche-Orient a indubitablement créé un climat propice à la fondation de sanctuaires isiaques au cours du III ${ }^{\mathrm{e}}$ siècle av. J.-C. Le cas des villes de la Tétrapole syrienne illustre ce phénomène, peut-être plus encore qu'on ne l'a affirmé jusqu'à présent, tout en attirant l'attention sur les conséquences religieuses de la troisième guerre de Syrie. En dehors du cas exceptionnel d'Antioche sur l'Oronte, où Ptolémée III Évergète serait à l'origine de l'institution du culte d'Isis, on insistera sur le rôle des fidèles venus d'Égypte dans les pas de l'armée lagide. L'établissement de ces derniers à Laodicée-sur-mer a permis d'assurer la pérennité du sanctuaire isiaque local jusqu'à l'époque romaine. La reconquête de la Syrie par les Séleucides, sans forcément faire table rase des fondations antérieures, comme on l'a vu à Séleucie de Piérie, semble en revanche s'être soldée par l'éclipse de Sarapis et d'Isis à

26 A. Houghton, The Early Seleucid Mint of Laodicea ad Mare (c. 300-246 вс), dans: M. Amandry, S. Hurter (éd.), Travaux de numismatique offerts à Georges Le Rider (Londres 1999) 169-184, en particulier 178-179.

27 Voir supra n. 21.

28 G. Le Rider, L'atelier de Posideion et les monnaies de la fouille de Bassit en Syrie, $B C H 110$ (1986) 406.

29 C.C. Lorber, The Ptolemaic Mint of Ras Ibn Hani, INR 2 (2007) 63-75; P.-L. Gatier, Héracléesur-mer et la géographie historique de la côte syrienne, Studi ellenistici 20 (2008) 269-283.

30 Les émissions monétaires séleucides ne reprennent sûrement à Laodicée-sur-mer que sous le règne d'Antiochos IV, vers 168-164 av. J.-C. Voir Seleucid Coins II, 75-76 n ${ }^{\text {os }}$ 14291431. Séries incertaines: Seleucid Coins I, 335 nº 926 (Séleucos III), 406-409 nºs 1069-1077 (Antiochos III) ; Seleucid Coins II, 662-663 no Ad152 (Séleucos II).

31 Cf., dans ce volume, la contribution de B. Legras, p. 95-115. 
Antioche. L'une des explications de ces fluctuations paraît résider, au ProcheOrient comme ailleurs, dans le rapport étroit, volontairement établi par les Ptolémées dès les débuts de leur dynastie et célébré tout au long de l'époque hellénistique, entre les cultes isiaques et le pouvoir royal.

\section{Abréviations (autres que celles de l'Année philologique)}

Bull.ép. Bulletin épigraphique de la Revue des études grecques (Paris 1888-).

IbIs J. Leclant et G. Clerc, Inventaire bibliographique des isiaca (Leyde 1972-1991).

ogis W. Dittenberger, Orientis graeci inscriptiones selectae (Leipzig 1903-1905).

RICIS L. Bricault, Recueil des inscriptions concernant les cultes isiaques (RICIS) (Paris 2005).

SEG Supplementum epigraphicum graecum (Leyde-Alphen aan den RijnAmsterdam 1923-).

Seleucid Coins I A. Houghton et C.C. Lorber, Seleucid Coins I (New York-LancasterLondres 2002).

Seleucid Coins II A. Houghton, C.C. Lorber et O.D. Hoover, Seleucid Coins II (New YorkLancaster-Londres 2008).

SNRIS

L. Bricault et al., Sylloge nummorum religionis isiacae et sarapiacae (sNRIS) (Paris 2008). 